\title{
Quantitative evaluation of blood on MRI
}

\author{
I. Gubskiy, D. Namestnikova, D. Nebesniy, L. Gubsky, V. Lelyuk, A. Osipov
}

Research Institute of Cerebrovascular Pathology and Stroke, Pirogov Russian National Research Medical University, Moscow, Russia Department of Fundamental and Clinical Neurology and Neurosurgery, Pirogov Russian National Research Medical University, Moscow, Russia Department of General and Medical Biophysics, Pirogov Russian National Research Medical University, Moscow, Russia

\section{Background and Aims}

Detection of blood on MRI is an important and relevant task for emergency radiology. Many factors can influence on visualization of blood, such as hemoglobin form, time after hemorrhage, presence of clot, pulse sequences and etc. The aim of this study was to create semi-automatically algorithm to define hemoglobin species on MRI.

\section{Method}

Special phantom with fixed temperature at magnetic field with 7T strength was used. Conventional MRI images and $\mathrm{T} 1, \mathrm{~T} 2, \mathrm{~T} 2 *$ maps and apparent diffusion coefficient (ADC) map was measured. Spectrophotometry was used to control of hemoglobin form and erythrocytes membrane status. Additionally, hemorrhage stroke was modeled in rat with dynamic MRI examination.

\section{Results}

Qualitative and quantitative MRI data of different determinations of blood (clot, free, plasma) total number of 15 was evaluated. In vivo MR in dynamics of rat with intracerebral hemorrhagic were obtained (from injection up to 42 day).

\section{Conclusion}

We have shown that semi-automatically algorithm trained on our data can detect different hemoglobin form in hemorrhagic stroke in rat. This method makes possible quantitative evaluation of blood on MRI, but still needs more improvements.

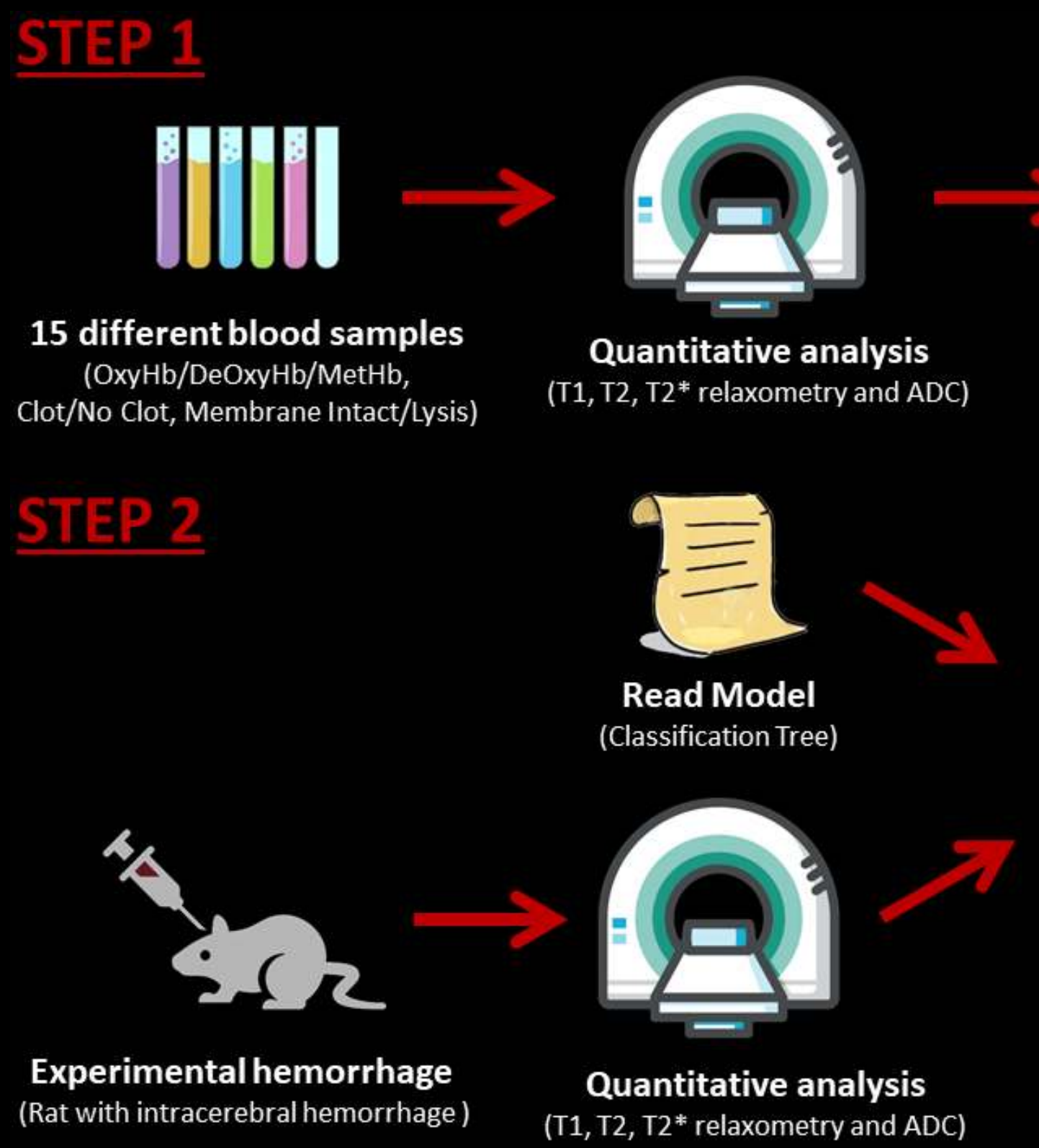

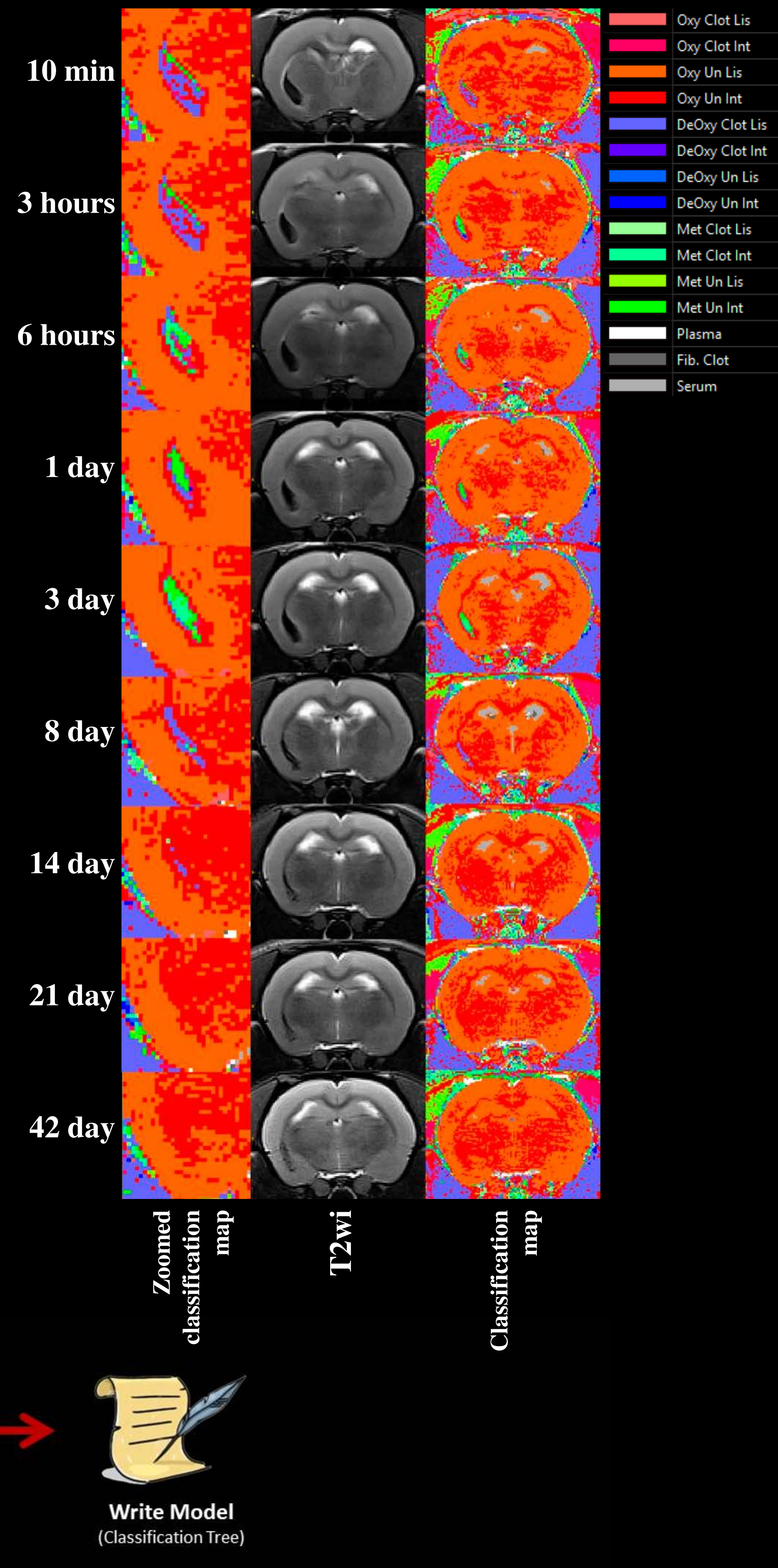

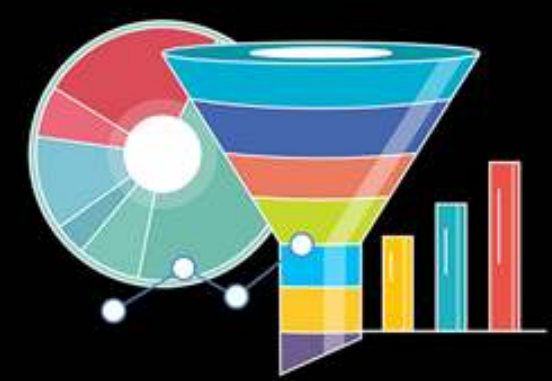

Automatic analysis (blood type calculation)

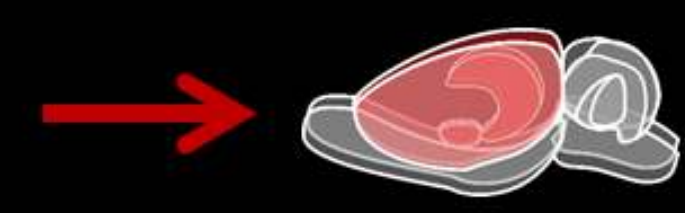

Classification maps of different blood types 